\title{
A traffic flow-oriented routing protocol for VANETS
}

\author{
Irshad A Abbasi ${ }^{1}$, Babar Nazir ${ }^{2}$, Aftab Abbasi ${ }^{3}$, Sardar M Bilal ${ }^{4}$ and Sajjad A Madani ${ }^{2}$
}

\begin{abstract}
This paper presents a novel position-based routing protocol for vehicular ad hoc networks (VANETs) to enhance traffic safety and traffic organization and facilitate driving through a smart transportation system. The protocol is referred to as the traffic flow-oriented routing (TFOR) protocol for VANETs. It considers a real-time urban scenario with multi-lane and bi-directional roads. It chooses junction optimally considering vehicular traffic flows to accomplish robust routing paths and thereby forwarding the data packets. The new junction selection mechanism and routing between the junctions is based on two-hop neighbor information, which increases packet-delivery ratio and decreases end-to-end delay. We designed, implemented, and compared TFOR against existing routing protocols of VANETs (greedy-perimeter stateless routing (GPSR), geographic source routing (GSR), and enhanced greedy traffic-aware routing (E-GYTAR)). Simulation outcomes in urban scenarios show that TFOR minimizes average end-to-end delay and routing overhead by on average $15.3 \%$ and $19.5 \%$, respectively, compared to GPSR. It reduces routing overhead up to $17 \%$ compared to GSR. TFOR maximizes packet-delivery ratio on an average of $17.5 \%, 10.7 \%$, and $7.2 \%$ compared to GPSR, GSR, and E-GyTAR, respectively.
\end{abstract}

Keywords: Traffic flow; Traffic organization; Vehicular ad hoc network (VANET); Position-based routing

\section{Introduction}

Recently, immense increase in the number of automobiles on the road made driving difficult and unsafe. Roads are routinely replete with vehicles, and therefore, safe-separation distance and sensible speeds do not seemed to be valued anymore. For instance, according to NHTSA (National Highway Traffic Safety Administration) [1] in 2006, there were an estimated 42,642 traffic-related casualties. The percentage (2\%) of casualties in 2005 was even higher than that of casualties in 2006. There are some advanced active and passive vehicle safety-related devices such as airbags, crumble zones, and anti-lock brakes invented to reduce causalities. The number of casualties in spite of all these latest devices has remained more than 40,000 per year for the last 15 years. Accidents do happen, but sight cannot be lost of the concept of road safety. Top car manufacturers and nationwide government agencies are determined to design solutions that assist drivers in anticipating danger and avoiding bad traffic zones. One such effort is the exploitation

\footnotetext{
* Correspondence: babarnazir@gmail.com

${ }^{2}$ Department of Computer Science, COMSATS Institute of Information

Technology (CIIT), Abbotabad 22060, Pakistan

Full list of author information is available at the end of the article
}

of wireless technology, to direct traffic issues, in the form of wireless access for vehicular environment (WAVE) devoted to the vehicular ad hoc networks (VANETs) [2-4]. The aim of WAVE is to overcome traffic issues and make driving efficient by giving timely guidance to drivers and vehicles that are not available through driver observations and independent sensors [1,5-7].

Vehicular communication is possible through either vehicle to vehicle $(\mathrm{V} 2 \mathrm{~V})$ or vehicle to infrastructure (V2I) or both. The goal of VANETs is to build an intelligent transportation system (ITS) [8]. It supports a wide variety of applications including prevention of accidents, traffic flow control mechanisms, information services, real-time alternate route computations, and provision of Internet ccess to the vehicles on motion [4,8-13]. Vehicular communication is an offshoot of mobile ad hoc network (MANET) $[9,12,14]$. VANETs have certain characteristics that change them from MANETs. In VANETs, >high-speed vehicular nodes have intermittent connectivity and they frequently change the network topologies. Physical factors restrict node movements and network topologies. The movement of vehicles is along roadways, and their mobility is restrained by traffic policies, such as traffic light signals, 
speed constraints, and road/traffic conditions. Power expenditure is not a vital concern in vehicular nodes, as vehicles can generate enough power to run the communication devices. In contrast, MANET's nodes are characterized by limited storage capacity, restricted battery power, low processing, and random movements with unpredictable mobility patterns $[8,9,12,13]$.

This work realizes the potential of VANETs to enhance traffic safety and traffic organization and to facilitate driving through a smart transportation system. In this work, we explored routing features of VANETs. We analyzed the previous routing literatures in VANETs. We have presented their contribution and limitations. By using the unique characteristics of VANETs, we have developed a novel position-based routing protocol called traffic floworiented routing (TFOR) protocol for VANET multi-lane roads. It considers the vehicle's position, direction, and speed to decide the junction and dispatch the data packets. It consists of two mechanisms: (1) new junction selection mechanism and (2) routing based on two-hop neighbor information. The new junction selection mechanism determines the directional and non-directional traffic flows based on real-time traffic in city environment. Determination of these flows provides shortest richdensity routing paths, which increase packet-delivery ratio and decrease end-to-end delay. We have also proved that forwarding, based on two-hop neighbor information, is a better choice than one-hop neighbor information, which also reduces the end-to-end delay and make routing more efficient. TFOR uses road topology and traffic density for efficient relaying of data in the network. It is useful for both delay-sensitive (like accident alerts, on-vehicle chat) and delay-tolerant (like infotainment) applications. The major contributions of this manuscript are as follows:

1. We provide a brief technical survey to analyze, compare, and present limitations of existing position-based routing protocols in VANETs.

2. We propose a novel position-based routing algorithm with new concepts of directional routing and non-directional routing to rout the packet through a shortest rich-density city road to increase packet-delivery ratio and decrease end-to-end delay and routing overhead.

3. We propose a forwarding technique based on two-hop neighbor information and its importance in the reduction of end-to-end delay to obtain optimum performance.

4. We implement, analyze, and compare the behavior of our approach with existing approaches (greedy-perimeter stateless routing (GPSR), geographic source routing (GSR), and enhanced greedy traffic-aware routing (E-GyTAR)) using VanetMobiSim and Glomosim simulators.
The rest of the paper is organized as follows. Section 2 describes the existing routing techniques along with their limitations. It also elaborates the motivation of the research work. Section 3 illustrates the proposed routing strategy. Analysis of the data and simulation outcomes are presented in Section 4. Finally, conclusions are presented in Section 5.

\section{Related work}

The available routing protocols in vehicular communication networks are broadly categorized into topology-based and position-based routing. The topology-based routing techniques can be reactive (on-demand), proactive (tabledriven), and hybrid. On-demand routing protocols (e.g., DSR [15], AODV [16]) maintain only those routing paths that are currently in use. While table-driven routing protocols (e.g., OLSR $[17,18]$ ) maintain all the available paths in the network topology. The maintenance of routing paths affects the performance of protocols in a highly dynamic network $[4,8,9,13,14]$. In position-based routing protocols, each vehicle contains a GPS receiver or other positioning abilities so that the vehicular nodes can have precise knowledge of their geological positions, movement directions, and speed. The location of destination node can be found using location services (e.g., HLS [19], GLS [20], and RLS [21]). There is no need of path maintenance $[4,8,11,22]$ as each node has to memorize its one-hop neighbors through beaconing. These characteristics of positionbased approach motivate us to focus on position-based routing for dealing the routing-related issues in VANETs. The existing position-based routing protocols are categorized as directional and non-directional. As the name suggests, the directional routing protocols $[10,23,24]$ focus on the direction of vehicles while routing the packets towards destination. The non-directional routing protocols $[4,8,11,14,22,25]$ do not focus on the direction of the vehicles while routing. Examples of both the categories are listed in Table 1.

A non-directional protocol is the greedy-perimeter stateless routing (GPSR) [14] that has been actually designed for highway scenarios. It works well in a highly dense network and operates in two phases, viz. greedy phase, and perimeter phase. In the greedy phase, a node sends the packet to one of its one-hop neighbors that is closest, among all the one-hop neighbors and of course the forwarding node itself, to the destination. If a node is having no one-hop neighbor that is closest to the destination than itself, the greedy phase meets the local maximum. Perimeter phase overcomes the local maximum situation. The perimeter phase consists of two modes: namely, the graph planarization and the right-hand rule. The perimeter phase causes delay in relaying the packet from the source to the destination [4] and may result in routing loops in the network. In addition, it cannot consider obstacles and, hence, shows poor performance in the city environment [4]. Furthermore, the 
Table 1 VANET routing protocols

\begin{tabular}{|c|c|c|c|c|c|c|c|c|c|c|}
\hline $\begin{array}{l}\text { Routing } \\
\text { protocols }\end{array}$ & Directional & Traffic-aware & Scenario & $\begin{array}{l}\text { Traffic data } \\
\text { required }\end{array}$ & $\begin{array}{l}\text { Carry and } \\
\text { forward }\end{array}$ & $\begin{array}{l}\text { Centralized/ } \\
\text { distributed }\end{array}$ & Environment & $\begin{array}{l}\text { Topology-based/ } \\
\text { position-based routing }\end{array}$ & $\begin{array}{l}\text { Forwarding } \\
\text { strategy }\end{array}$ & $\begin{array}{l}\text { Routing table } \\
\text { based on one- hop } \\
\text { neighbor information }\end{array}$ \\
\hline \multirow[t]{2}{*}{ 1. GPSR [14] } & $N$ & $N$ & Highway & No & $N$ & Distributed & Dense & Position & Greedy & $Y$ \\
\hline & & & & & & & & & Forwarding & \\
\hline \multirow[t]{2}{*}{ 2. GSR [4] } & $N$ & N & City & No & N & Distributed & Sparse & Position & Greedy & Y \\
\hline & & & & & & & & & Forwarding & \\
\hline \multirow[t]{3}{*}{ 3. GPCR [8] } & $N$ & N & City & No & N & Distributed & Sparse & Position & Restricted & Y \\
\hline & & & & & & & & & Greedy & \\
\hline & & & & & & & & & Forwarding & \\
\hline \multirow[t]{2}{*}{ 4. A-STAR [22] } & $N$ & Y & City & No & Y & Distributed & Sparse & Position & Greedy & Y \\
\hline & & & & & & & & & Forwarding & \\
\hline \multirow[t]{3}{*}{ 5. GyTAR [25] } & $N$ & Y & City & Yes & Y & Distributed & Both & Position & Improve & Y \\
\hline & & & & & & & & & Greedy & \\
\hline & & & & & & & & & Forwarding & \\
\hline \multirow[t]{2}{*}{ 6. DGR [24] } & Y & N & Highway & No & Y & Distributed & Both & Position & Greedy & Y \\
\hline & & & & & & & & & Forwarding & \\
\hline \multirow[t]{2}{*}{ 7. PDGR [10] } & Y & N & Highway & No & Y & Distributed & Both & Position & Greedy & Y \\
\hline & & & & & & & & & Forwarding & \\
\hline \multirow[t]{2}{*}{ 8. RDGR [10] } & Y & N & Highway & No & Y & Distributed & Both & Position & Greedy & Y \\
\hline & & & & & & & & & Forwarding & \\
\hline \multirow[t]{2}{*}{ 9. PDVR [23] } & Y & N & Highway & No & Y & Distributed & Sparse & Position & Directional & Y \\
\hline & & & & & & & & & Forwarding & \\
\hline \multirow[t]{3}{*}{ 10. E-GyTAR [26] } & Y & Y & City & Yes & Y & Distributed & Both & Position & Improve & Y \\
\hline & & & & & & & & & Greedy & \\
\hline & & & & & & & & & Forwarding & \\
\hline
\end{tabular}


graph planarization fails in city scenarios and may cause partitioning of the network due to obstacles. Last but not the least, the fact is that GPSR is not a traffic-aware routing protocol [22].

One of the first attempts to handle the routing issues in city scenarios was the geographic source routing (GSR) [4] which employs the position-based knowledge with the topological knowledge of the network. It runs Dijkstra's algorithm to locate the shortest route connecting the source and the destination. GSR computes a sequence of junctions based on the shortest route from source to destination using a street map that packets must have to traverse. It employs greedy forwarding to dispatch the data packets from source to target node. The greedy forwarding approach, as already stated, has the tendency to stick onto a local maximum. In this, eventuality, GSR uses a carry-and-forward approach as a recovery strategy. This protocol does not consider the number of vehicles between the junctions before forwarding packet. Endto-end connection is difficult in case of low traffic density along a preselected path [25]. It degrades the performance of GSR. In addition, it is not a traffic-aware routing protocol.

As against GSR, the greedy-perimeter coordinator routing (GPCR) [8] is a map-independent routing technique. It includes restricted greedy forwarding and repair strategy. Restricted greedy forwarding prefers to choose the coordinator (the node on a junction) over a non-coordinator node when deciding the next hop, even if the former is not the geographically nearest node to destination. The routing decision is, thus, made at the coordinator node that decides the street the packet should follow next. The repair strategy overcomes the local optimum problem. It consists of a perimeter mode without any graph planarization phase. It is assumed that the graph planarization is natural in a city environment. So, there is no need of computing graph planarization, because it may cause a partitioning in the network. Similar to GSR, the GPCR neglects low traffic density case. Furthermore, it is not a traffic-aware routing protocol.

The anchor-based street and traffic-aware routing (ASTAR) is a traffic-aware routing protocol [25], as opposed to GPSR and GSR. From these latter two protocols, A-STAR has two main peculiarities. Firstly, it uses a statically or dynamically rated map for traffic awareness and uses these maps to identify paths having higher number of vehicles. Secondly, A-STAR has a new local recovery technique, considered to be better than those of GSR and GPSR [22], for the packets got stuck in local maximum. The path selected by A-STAR on the basis of anchors may not be the shortest, due to which it may have higher end-to-end delay $[4,24]$.

Designed for city environment, the greedy traffic-aware routing protocol (GyTAR) [25] selects the junctions dynamically as against the static approach of GSR and A-STAR. GyTAR exhibits three mechanisms: (i) a completely decentralized scheme, named the infrastructure free traffic information system (IFTIS) [21], which estimates traffic density between the junctions in the urban roads; (ii) a mechanism for dynamic junction selection, i.e., when deciding the next destination junction, the source vehicle, or an intermediate vehicle in a junction finds the position of the neighboring junctions using the map, which allocates a score to each neighboring junction assuming the curve metric distance to the destination and the traffic density; and the next junction is the one that has highest traffic density and closest to destination vehicle, (iii) which applies an improved greedy forwarding mechanism to forward the packet between the two involved junctions. The wrong junction selection mechanism compels the packet to get stuck onto local maximum, especially at low traffic density or when all the vehicles moved away from the destination on the selected street, which is its failure [26]. The directional routing protocols [4,8,11,14,22,25] focus on the direction of the vehicles while routing. The technical detail of these routing protocols is presented below. The main problem with the non-directional routing protocols is the routing loop formation while routing the packet, which may cause delay [10]. Sometimes, the packet is sent to a vehicle that is moving away from the destination, resulting in packet loss.

Directional greedy routing protocol (DGR) [10] resolves the issues outlined above by taking into consideration the direction of the vehicle and assigns higher weight to the vehicle that is moving towards the destination. It uses a carry-and-forward approach when packets get stuck onto a local maximum and works well in a highway scenario.

Predictive directional greedy routing protocol (PDGR) is an extended version of DGR that employs a predictive compared to the latter which only takes into account the current neighbors while calculating the weighted score. PDGR determines the weighted score for the packet carrier, its present neighbors, and the possible expected neighbors in the very near future. It decides the next hop based on these weighted scores. The packet carrier obtains the information of possible future neighbor based on the two-hop neighbor information. This information is achieved through periodical sending of hello messages. The use of a prediction mechanism makes the PDGR outperform the DGR in terms of end-to-end delay and delivery ratio.

The reliable directional greedy routing protocol (RDGR) [27] minimizes link breaks, improves route reliability, and enhances packet-delivery ratio. In DGR and PDGR, the likelihood of packet loss increases if the neighbor node moving in the direction of destination has higher speed as compared to source node or intermediate forwarder node. RDGR enhances DGR by introducing the new metric of link stability. In RDGR, a source node or an intermediate forwarding node chooses the next neighbor node having a higher speed as well as a stable link.

The position-based directional vehicular routing (PDVR) protocol deals with straight and curvy highway roads [23]. It 
selects stable and efficient route for routing packet to the destination based on two rules. First, the neighbor selected for forwarding packet should move in the same direction as the source or the intermediate packetforwarding node. Secondly, its direction must be similar to that of the destination. PDVR may not work well in a city environment because of some obstacles. It is not a traffic-aware routing protocol.

The DGR, PDGR, and RDGR protocols take into account only the highway scenarios [27]. The enhanced greedy traffic-aware routing (E-GyTAR) protocol [26] selects a routing path by using an enhanced junction selection mechanism. In this mechanism, the vehicular node on the current intersection selects the next intersection considering the number of vehicles that are moving in the direction of the destination. If there is completely an opposite flow of the vehicles and none is moving toward destination, then it cannot select a routing path that may reduce packet-delivery ratio and enhance end-to-end delay.

In general, most of the above routing approaches are not traffic aware. As a result, they forward the packets along the city street where moving vehicles are absent. In such situation, packets meet local optimum and are discarded. These problems can be solved by having a mechanism that provides timely information about traffic on the city streets. Some of these routing protocols (like A-STAR, E-GyTAR) are traffic aware but they are unable to use real-time vehicular traffic density properly. As a result, these are inefficient in routing. In between successive junction, most of the above routing protocols (GSR, GPSR, A-STAR, etc.) use simple greedy forwarding. However, simple greedy does not consider neighbors' speed and direction. In addition, it uses only one-hop neighbor information. Hence, it misses some suitable candidate vehicles for packet forwarding.
We propose a routing protocol that presents a solution to the aforementioned problems. The protocol accomplishes robust routes within urban environment. The protocol envisioned to work well for different types of VANET applications and ensure user connectivity. These applications include road-safety services (like traffic flow control mechanism, issuing driving alerts like traffic jams, accident warnings, road's condition, etc.) and comfort services (like gas station location, Internet access, music downloading, games, etc.).

\section{TFOR}

\subsection{Problem formulation}

Figure 1 explains the scenario where E-GyTAR does not work well. The source vehicle $S$ on the current junction wants to send data packets to the destination vehicle D. E-GyTAR selects the next neighbor junction considering the number of vehicles that are moving in the direction of the destination. In this scenario, no vehicle is moving towards the destination. All the vehicles are moving in the opposite direction of the destination but provide enough connectivity between S and D. E-GyTAR will not be able to select the junction $\mathrm{J} 2$ that is the closest junction to the destination. When the network is sparse, E-GyTAR performs well. However, when the numbers of vehicles are increased, a point comes where there is an adequate number of vehicles moving in an opposite direction and providing connectivity. Hence, E-GyTAR cannot find the geographically closest path towards destination. This reduces packetdelivery ratio and enhances end-to-end delay. Therefore, we need a routing protocol that can select the routing paths/junctions based on directional density and otherwise. To tackle this issue, this paper suggests novel routing protocol called the traffic flow-oriented routing (TFOR) protocol.

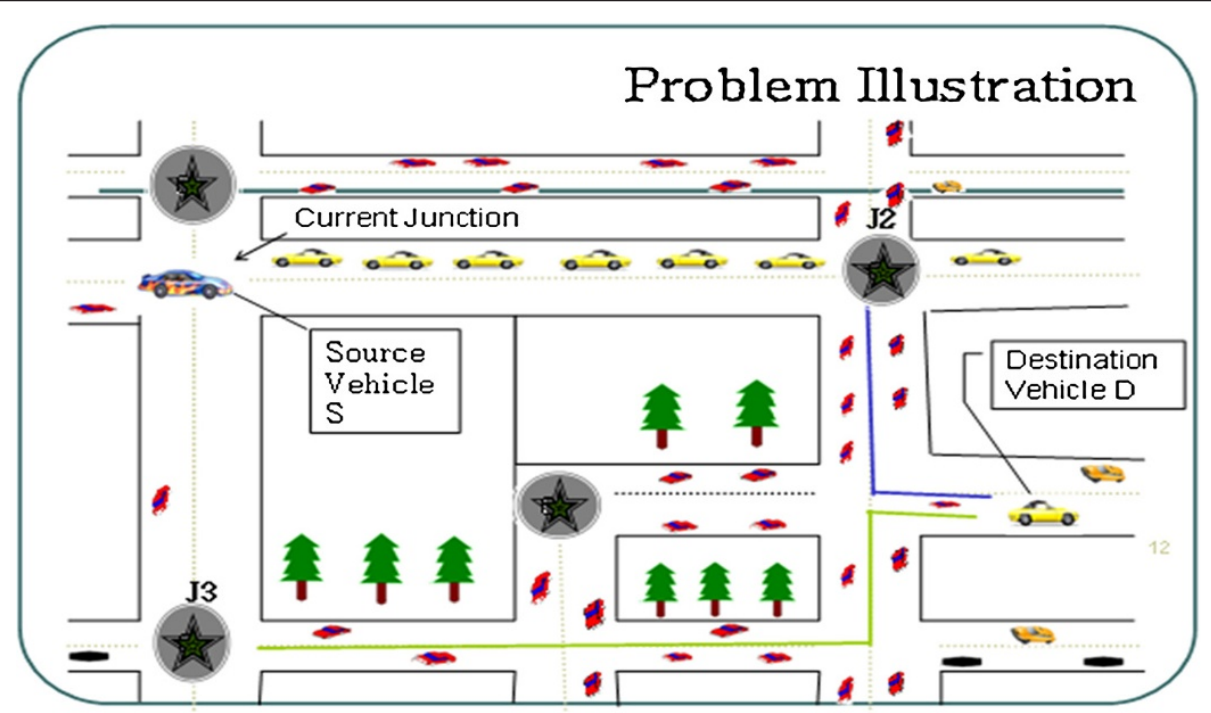

Figure 1 The problem scenario. 


\subsection{TFOR protocol}

TFRO is a junction-based geographical routing protocol, competent to accomplish robust routes, considered to exhibit optimal performance in city scenarios. To attain this goal, it employs GPS to locate its own position. It uses grid location service (GLS) to acquire the position of destination vehicle [20]. Every vehicle contains on-board navigation system that finds out the location of neighboring junctions and gives valuable city's street information with preloaded digital maps. It contains two mechanisms, viz., junction selection mechanism, and routing between junctions based on two-hop neighbor information.

\subsubsection{The junction selection mechanism}

Unlike E-GyTAR, which ignores non-directional density with respect to destination, TFOR selects the junctions dynamically considering directional as well as non-directional flow of traffic density. The probability of occurrence of directional and non-directional traffic with respect to certain destinations in urban multi-lane road is equally likely. Therefore, directional and non-directional traffic is equally important for connectivity provision in VANETs. Ignoring a part of traffic density means neglecting connectivity. Compromise on connectivity degrades performance of ad hoc networks. The sending vehicular node in a junction uses a digital map to identify the location of neighboring junction. Location service makes the position of destination vehicle available. The sending vehicle after that determines the curvemetric distance from each neighbor junction to the destination vehicle. It uses algorithm 1 to assign a score to each neighbor junction. It chooses the junction with maximum score as a next destination junction. The chosen junction is the nearest to the destination vehicle. It has also the higher traffic density flow.
In algorithm 1, the following equation calculates the score that determines the next destination junction $(\mathrm{N} \mathrm{Dj})$.

$$
\text { score }=\alpha \times\left(1-D_{p}\right)+\beta \times \mathrm{TD}
$$

where $D_{p}=D_{n} / D_{c}$ decides the nearness of neighbor junction to the destination point.TD represents the total traffic density between the current junction $\left(C_{\mathrm{j}}\right)$ and the neighbor or next-candidate junction $\left(\mathrm{N} \mathrm{C}_{\mathrm{j}}\right)$, may be directional or non-directional. $\alpha$ and $\beta$ are the weight factors, such that $\alpha+\beta=1$.

With equal importance of the two factors, $\alpha$ and $\beta$ are both set to the value 0.5 . Equation refeq:score suggests that the junction that has maximum score is chosen as the next destination junction. It is also geographically the nearest junction to the destination. As revealed in Figure 1, the proposed junction selection mechanism selects J2 instead of selecting J3 as the next destination junction because of the shortest distance and vehicular traffic density (directional or non-directional). Afterwards, the TFOR junction selection mechanism chooses the best available shortest path to the destination considering the vehicular density.

TFOR uses the infrastructure-free traffic information system (IFTIS) [20] to calculate traffic density between pairs of junctions. For our scenario, the road between the two junctions is divided into small cells (circles) of fixed size as shown in Figure 2. The vehicles belong to the same cell from a group. The cell size is equivalent to the vehicle's transmission range that is around $250 \mathrm{~m}$. The cells superimpose in such a way that each vehicle belongs to at least one of the cells. Any vehicle that is nearest to the cell's center is named as the group head/leader, for the duration is

Algorithm l: The junction selection mechanism

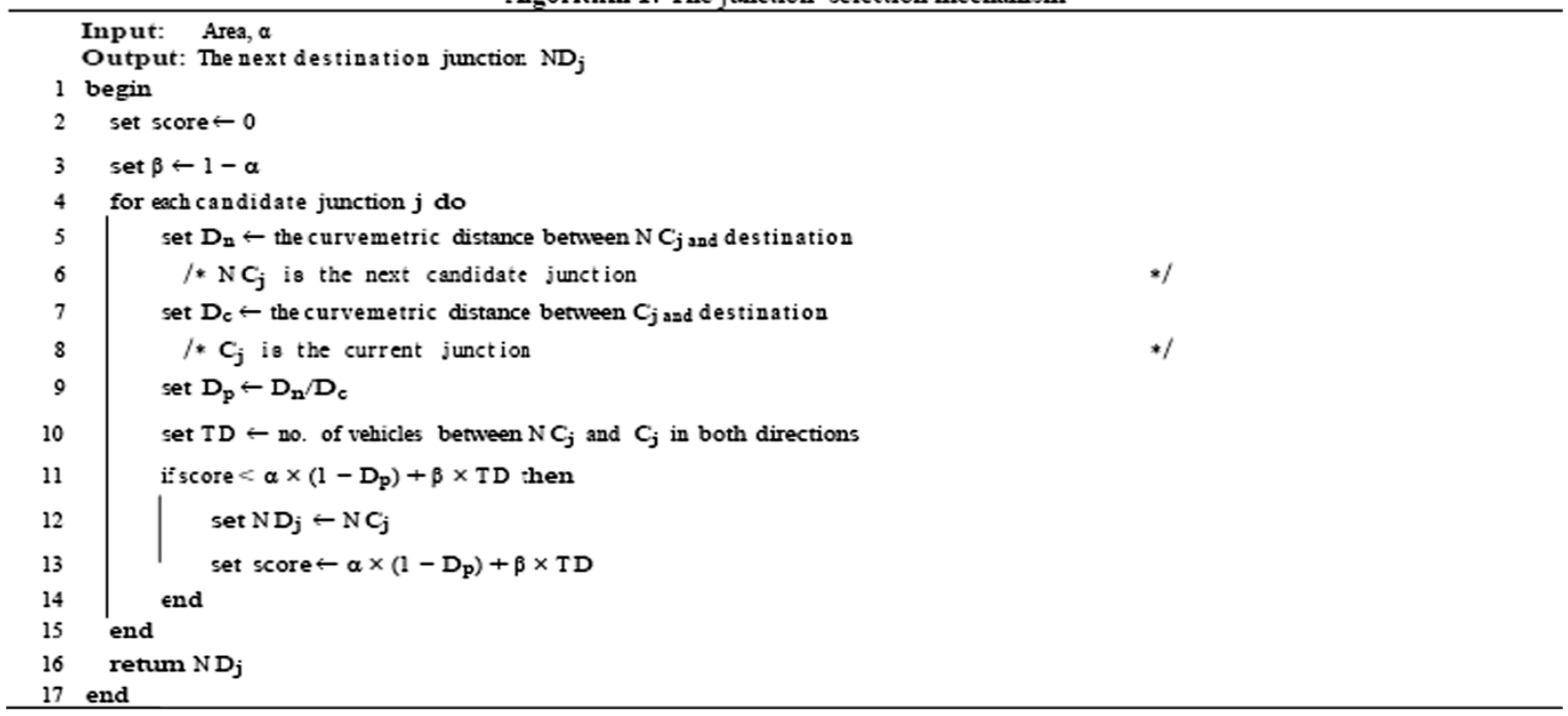




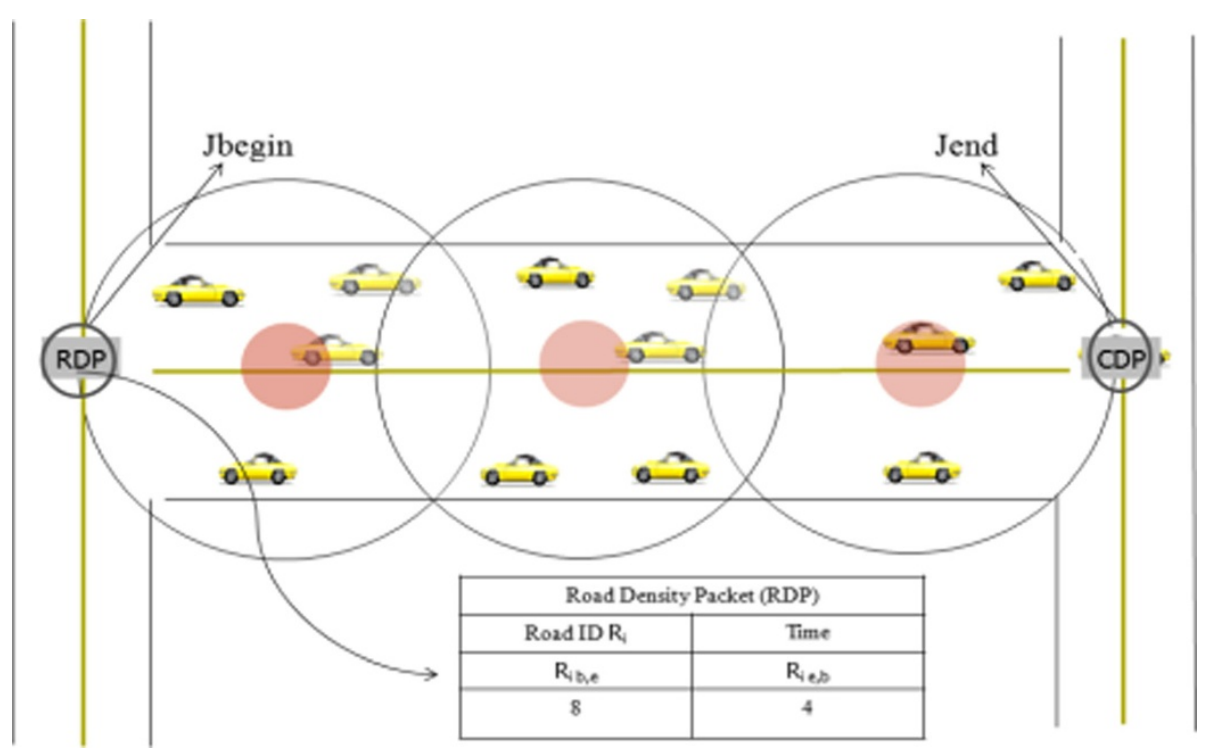

Figure 2 Road density packet.

the closest to the center. The cell's center locations are being represented by tiny red circles called red zones. Only the group head generates and updates the cell density packet (CDP) by including the entire vehicles (the number of vehicles moving in the direction of destination and the number of vehicles moving opposite to destination) present inside the cell. The vehicle, to depart the road, initiates the CDP. The CDP contains the road ID,the transmission time, the list of cell-center positions also called route anchors, the total density of a cell, and the directional as well as the non-directional density of the traffic within the cell. The CDP's data format is illustrated in Figure 3. Packets travel along the cell centers to reach other intersections. The whole density estimation method is shown in Figure 2.

Algorithm 2 enlightens the procedure of the packetforwarding mechanism that follows the following steps.

- The node F (forwarding vehicle) forwards the CDP packet between two junctions (i.e., Jend $J_{\text {begin }}$ ) of the road section. It forwards the CDP packet from the end of junction $J_{\text {end }}$ to the beginning of junction $\mathrm{J}_{\text {begin }}$.
- F may or may not be the group head. If it is a group head, then it will consult its neighbor table and update the CDP packet by adding the total density $|\mathrm{V}|_{\text {itotal }}$ of the cell to which it belongs, directional density $|\mathrm{V}|_{\mathrm{i}} \uparrow$, and the non-directional density $|\mathrm{V}|_{\mathrm{i} \downarrow} \downarrow$. $F$ then forwards the CDP packet to the vehicular node that is nearest to the next anchor and so on.

- When the CDP packet reaches the group head of the last anchor, it updates the packet and estimates the mean and variance related to the cell density. After that, estimated density will be forwarded to the destination junction.

- If forwarding vehicle $\mathrm{F}$ has no appropriate neighbor vehicle closer to the next anchor, it will keep the CDP until it locates a vehicle that is nearest to the next anchor.

- When the other junction $\left(\mathrm{J}_{\text {begin }}\right)$ is reached, the CDP is converted into a road density packet (RDP) and it is propagated around the junction.

Note that the number of vehicles within cell i moving from the start of junction to the end is represented by $|\mathrm{V}|_{\mathrm{i}} \uparrow$, whereas $|\mathrm{V}|_{\mathrm{i} \downarrow}$ indicates the vehicles in cell i moving from end of junction to the start of junction.

\begin{tabular}{|l|l|l|}
\hline \multicolumn{4}{|c|}{ Cell Data Packet(CDP) } \\
\hline Road ID & \multicolumn{1}{|c|}{ Cell' ID } \\
\hline Direction & Cell's Center Position & Cell's Total density \\
\hline Time & Cell directional density & Cell's Non-directional density \\
\hline
\end{tabular}

Figure 3 The cell density data packet format. 


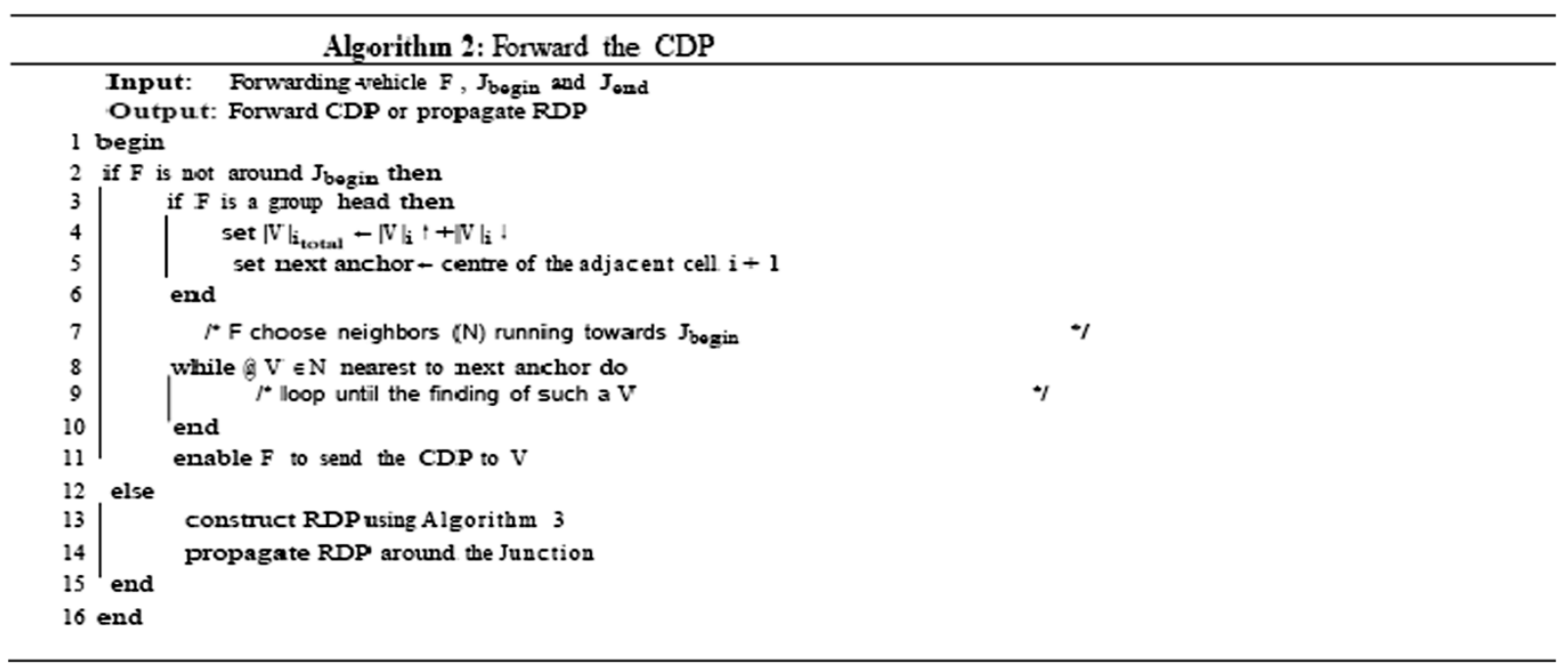

The RDP construction is explained in algorithm 3. In this algorithm, for a road $i$, if the forward direction road density $\left(R_{i} \uparrow\right.$ or $\left.R_{i b, e}\right)$ is not available, then the non-directional density $\left(R_{i} \downarrow\right.$ or $\left.R_{i e, b}\right)$ should be considered for routing and vice versa. If both are available, then the flow ratio $\gamma$ of the directional to non-directional density is determined. If $\gamma \geq 1$, then the forward density is greater and the forward route is preferred; otherwise, the back route is considered. Thus, all the vehicles around the junction are acquainted with total road density $\left(R_{\text {itotal }}\right)$ in both directions. In this manner, TFOR estimates the road traffic density.

\subsubsection{Routing between junctions}

After the determination of destination junction, TFOR exploits two-hop neighbor information with greedy approach for forwarding data packets between two concerned junctions. For that purpose, it marks all data packets with the position of the next junction. Every vehicle keeps up a neighbor table that records the position, direction, and speed of each neighbor vehicle. Periodical beacon messages update the entries of neighbor table. Before forwarding data packet, source or intermediate vehicle consults its neighbor table for latest anticipated positions of neighboring vehicles.

GyTAR and E-GyTAR are using predictive strategy based on one-hop neighbor information. According to

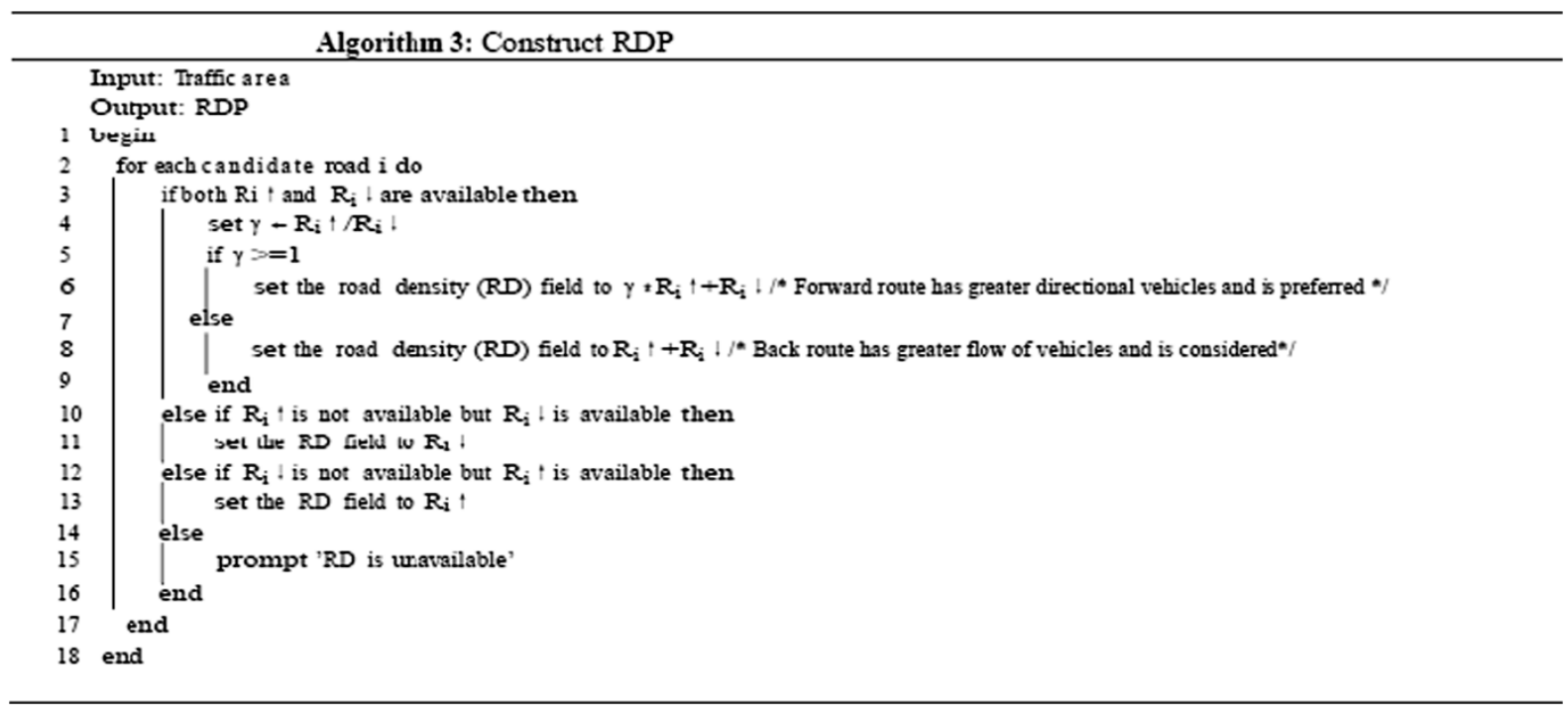




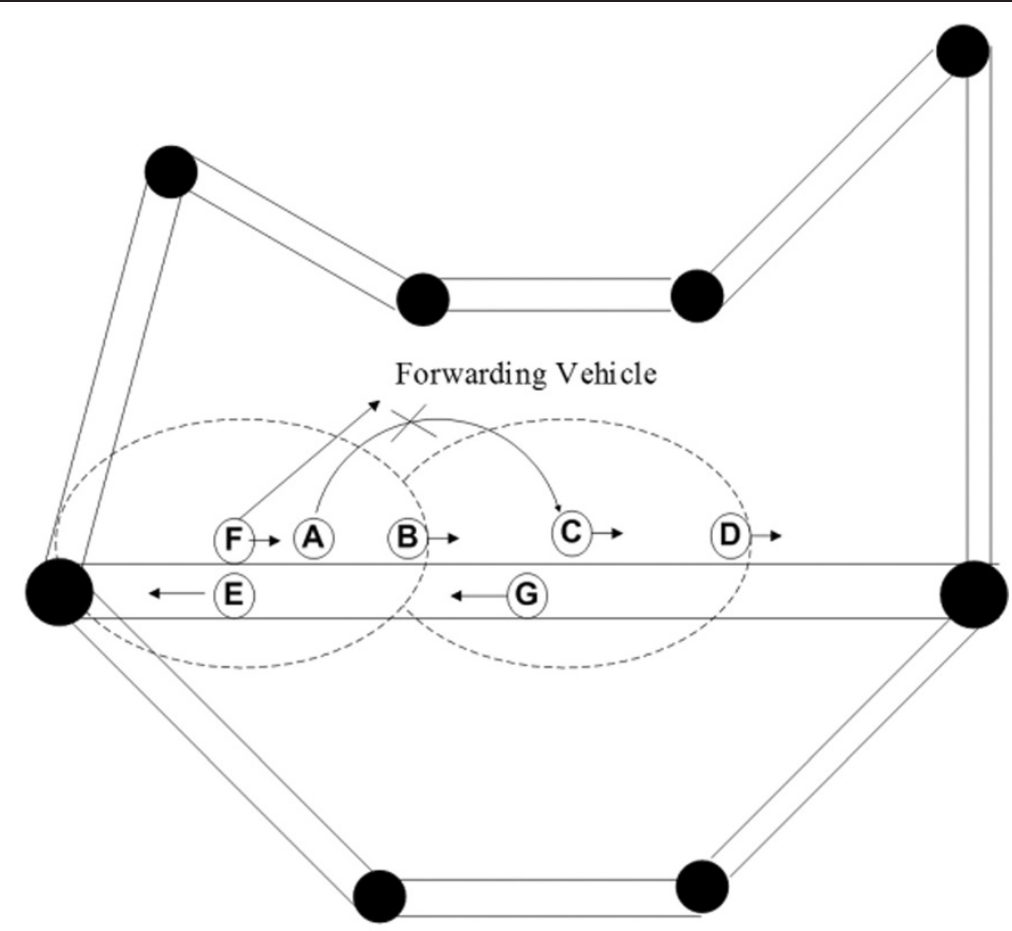

Vehicular Node

Two way road

Intersection

Figure 4 Routing between junctions.

that predictive strategy in Figure 4, the forwarding vehicle $\mathrm{F}$ sends the packet at time $\mathrm{t} 1$ to vehicle $\mathrm{A}$ because it has greater speed than vehicle B. At time $\mathrm{t} 2$, A cannot forward the packet to vehicle $C$, because it is outside the range of vehicle $\mathrm{C}$. But, If $\mathrm{F}$ uses two-hop neighbor information which is achieved by beacon messages, then $\mathrm{F}$ will dispatch the packet to vehicle $\mathrm{C}$ at time $\mathrm{t} 2$ through neighbor B instead of vehicle A. It is because B is closer to $C$ and $C$ is in turn the closest vehicle to destination D. This minimizes the end-to-end delay further and diminishes the number of hop counts as well, which makes the routing more efficient. TFOR is based on two-hop neighbor information instead of one hop. Every node maintains two-hop neighbor's table by exchanging

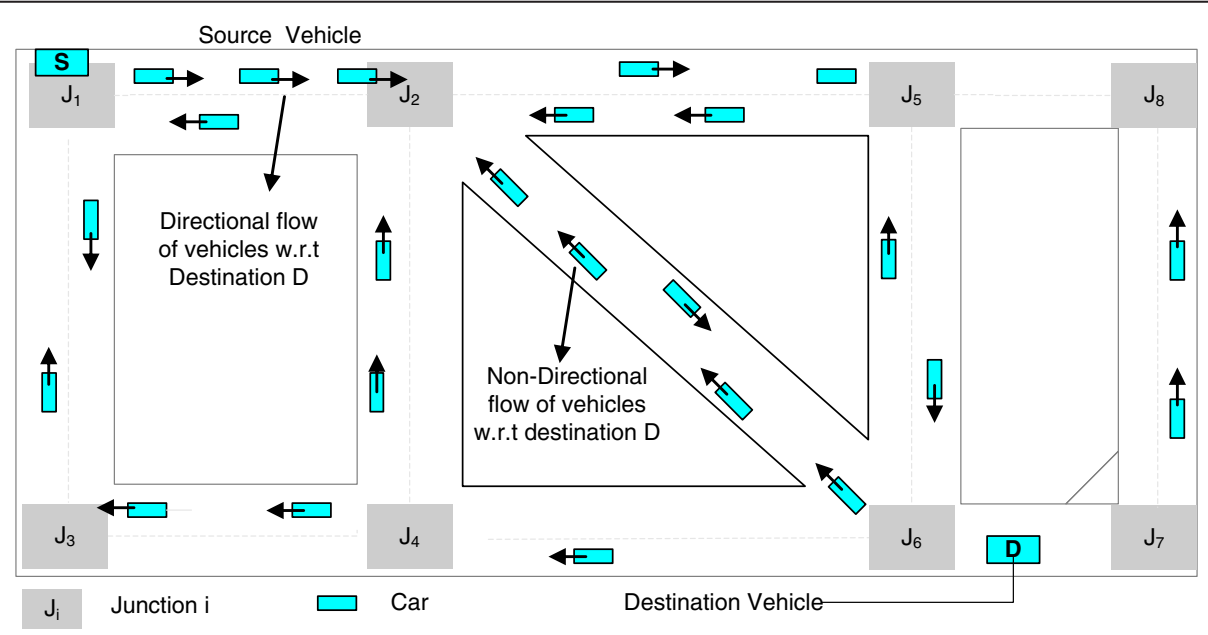

Figure 5 Working of TFOR. 


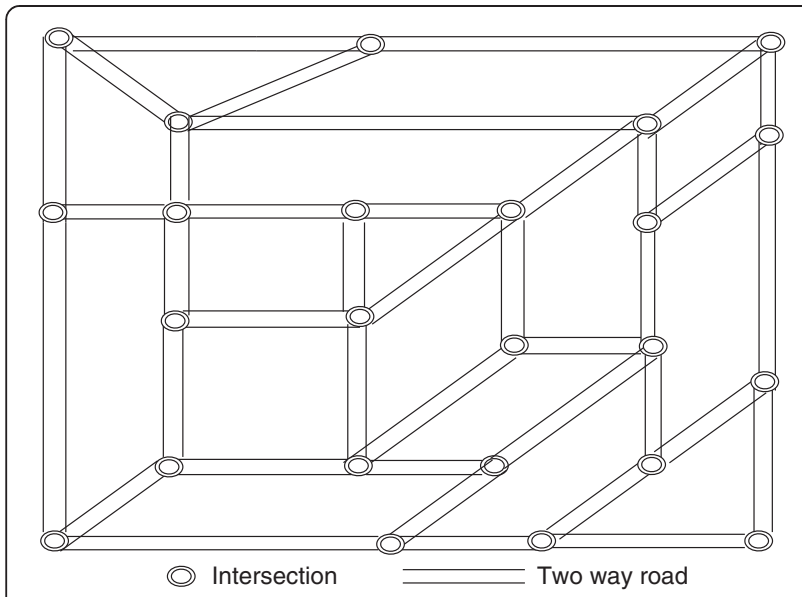

Figure 6 The city simulation area.

one-hop neighbor entries through beaconing. The forwarding node or source node looks its two-hop neighbor table and uses greedy forwarding to dispatch the packet through its neighbor to the neighbor's neighbor that is closest to destination. TFOR uses the carry-and-forward technique of [22] to recover from the local optimum situation.

\subsection{An illustrative example}

In Figure 5, the source vehicle $\mathrm{S}$ sends data packet to destination vehicle D. Source or forwarding vehicle at current junction $\mathrm{J} 1$ uses algorithm 1 to compute the next candidate junction based on the traffic flow and the shortest distance to the destination. Roads between the junctions are two lanes. Some lanes are rich in directional and some are rich in non-directional traffic flows with respect to destination. These flows provide enough connectivity for sending packet towards destination. Algorithm 2 and algorithm 3 are used to compute directional and non-directional traffic flows. Source vehicle at current junction $\mathrm{J} 1$ computes scores for neighbor junctions. According to algorithm 1, J2 has higher score as compared to J3 because of the shortest distance to the destination and higher traffic density. Therefore, it selects $\mathrm{J} 2$ as the next candidate junction instead of J3 because $\mathrm{J} 2$ is the closest to destination and has higher traffic directional flow as compared to J3. At J2, source vehicle assigns a score to J4, J5, and J6. It selects J6, as it has a higher score as compared to J4 and J5 because of the shortest distance and higher non-directional flow of traffic. This process continues until packet reaches the destination. Between the junctions, two-hop neighbor information is used as packet-forwarding mechanism that is already described in Section 3.2.2.

\section{Simulation results}

\subsection{Simulation setup}

To determine the performance of proposed routing protocol (TFOR), we carried out simulations in GLOMOSIM. The simulations pertaining to a city scenario, an existing directional protocol (E-GyTAR), and two non-directional routing protocols (GPSR and GSR) for performance comparison were selected. The mobility model [28] for VANETs' simulation should reflect the actual properties and activities of vehicles. There are two categories of mobility models, namely, the macroscopic models and microscopic models. The macroscopic model includes mobility limitations such as speed restrictions, urban streets, roads, traffic lights, traffic flows, number of lanes, and traffic density. The microscopic model determines vehicle activities with respect to other vehicles as well as the underlying infrastructure. We have chosen VanetMobiSim model [28], which can provide both macro and micro mobility. It is an enhancement of the CANU mobility simulation environment [2] and is suitable for VANETs.

In our simulation, the VanetMobiSim generates vehicular mobility patterns, which simulate a 3,000 $\times$ $2,900 \mathrm{~m}^{2}$ area. The city simulation area consists of 38 bi-directional roads (with multi-lanes) and 24 intersections, as shown in Figure 6. The vehicular nodes are disseminated randomly above the roads and initiate movements in both directions. The intelligent-driver model [23] manages the movements of vehicular nodes on the roads. Table 2 summaries all the essential simulation parameters. The simulation outcomes revealed below are the average of several simulation runs.

Table 2 The simulation parameters

\begin{tabular}{lll}
\hline Simulation/scenario & & MAC/routing \\
\hline Simulation time & $200 \mathrm{~s}$ & MAC protocol \\
Map size & $3,000 \times 2,900 \mathrm{~m}^{2}$ & Channel capacity \\
Mobility model & VanetMobiSim & Transmission range \\
Number of intersections & 24 & Traffic model \\
Number of roads & 38 & Packet sending rate \\
Number of vehicles & 75 to 250 & Weight factors $(a, \beta)$ \\
Vehicle speed & $35 \mathrm{t} \mathrm{o} 60 \mathrm{~km} / \mathrm{h}$ & Packet size
\end{tabular}




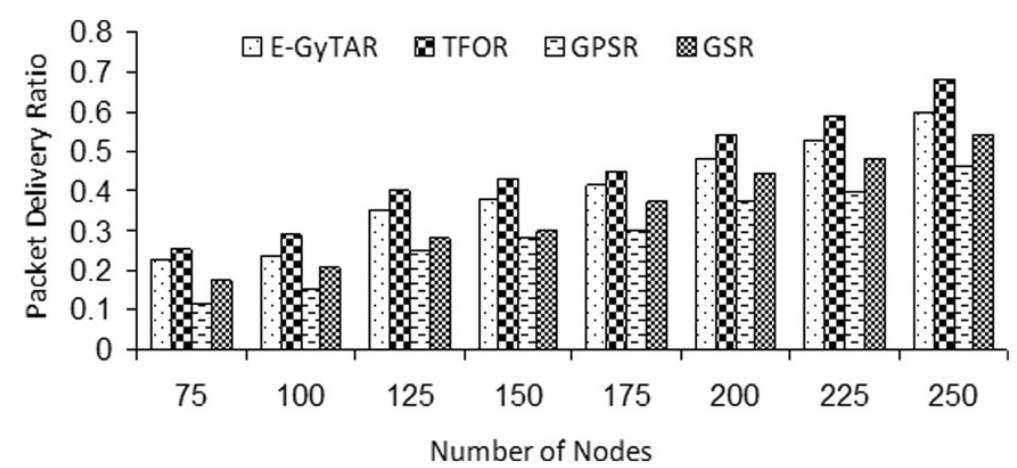

Figure 7 Delivery ratio as a function of the number of nodes (at 5 packets/s).

\subsection{Results}

Delivery ratio, end-to-end delay, and routing overhead [19] were selected as evaluation metrics. Based on these metrics, the performance of the selected protocols is being discussed below.

\subsubsection{Packet-delivery ratio}

Packet-delivery ratio is the ratio of data packets successfully received at the destination to those produced by the source [24]. Figure 7 reveals the consequences of growing network-traffic density on the packet-delivery ratio with fixed CBR rate. GPSR, GSR, and E-GyTAR exhibit less delivery ratio than TFOR. In GPSR, when greedy forwarding meets the local optimum, then it uses perimeter mode in order to come out of the local optimum situation. In perimeter, the node has a less opportunity to send the packet to a node approaching it in near future. In addition, the perimeter mode uses graph planarization phase that causes partition even in the connected network at the junctions in city environment, which reduces the packet-delivery ratio. GSR does not consider traffic density while selecting junctions statically based on a city street map; some streets may not contain enough vehicles to route the packets towards the destination, leading to low packet-delivery ratio. In E-GyTAR, the junction selection mechanism is solely based on directional vehicles; in the absence of directional vehicles, it meets local maximum and misses the non-directional vehicles that are available for routing as depicted in Figure 7. This results in reduction of packetdelivery ratio. In TFOR, the dynamic junction selection mechanism uses both directional and non-directional traffic density depending on their availability and shortest path to the destination. It uses both the paths efficiently which contributes to the packet-delivery ratio. As depicted in Figure 7, the two-hop neighbor information is a better choice for deciding the next hop that is closest to destination; it also enhanced the packet-delivery ratio of TFOR. The packet-delivery ratio of all the protocols increases as the network density becomes high because connectivity increases.

Figure 8 plots the packet-delivery ratio against the packet-sending rate. The packet-delivery ratio decreases if $\mathrm{CBR}$ rates are increased to a certain value. It is because this process induces congestion and so the network drops certain packets. The perimeter phase in GPSR causes more packet transmission. Hence, high packet sending rate causes decrease in packet-delivery

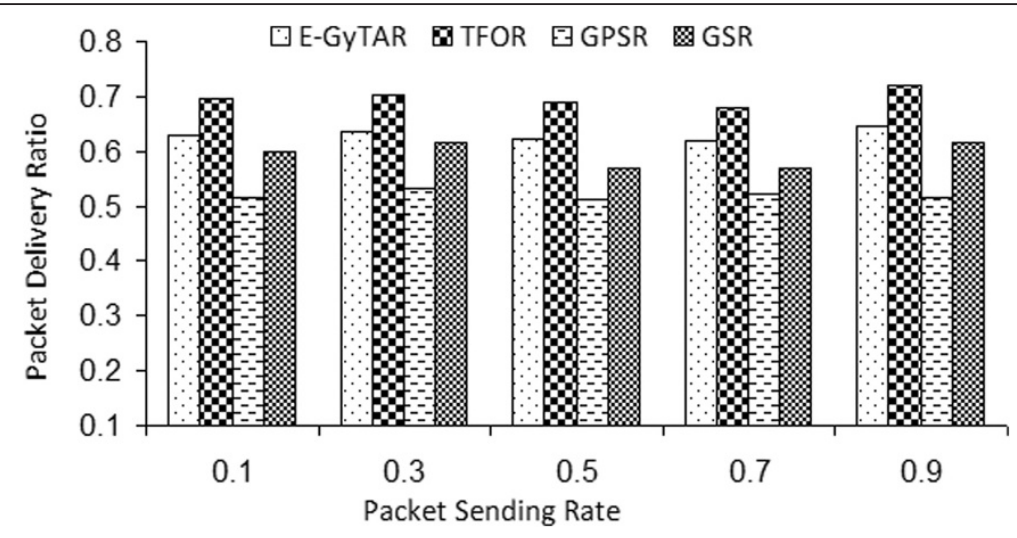

Figure 8 Delivery ratio as a function of the packet sending rate (225 nodes). 


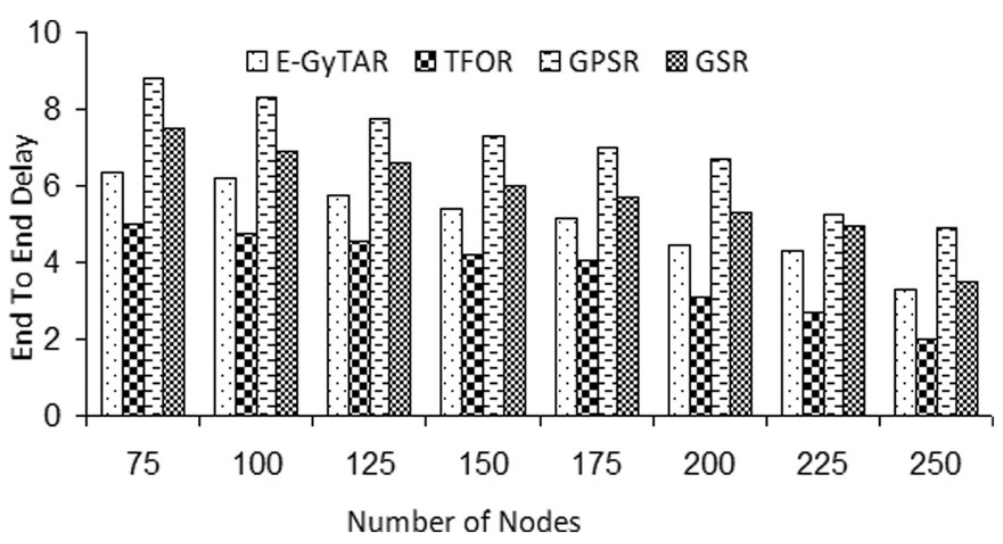

Figure 9 End-to-end delay as a function of the number of nodes (at 5 packets/s).

ratio of GPSR. In GSR, some nodes along the preselected paths cause more packet transmission. This causes reduction in packet-delivery ratio because of high packet sending rate. For E-GyTAR and TFOR, the packet-sending rate will not spoil their performance to much in terms of packet-delivery ratio.

\subsubsection{End-to-end delay}

The end-to-end delay is the average time taken for a packet to pass through the network from its origin to destination [24]. Figure 9 shows that the end-to-end delay of GPSR is more than that of GSR, E-GyTAR, and TFOR. This is because these three approaches employ carry-and-forward approach as an alternative to perimeter phase of GPSR. Carry-and-forward approach provides more opportunity to locate some appropriate incoming node for forwarding occasionally. The perimeter phase of GPSR misses this chance. Even in the connected network, the perimeter phase with planner graph induces disconnections in the network. It causes delay in relaying the packet from source to destination. When the density of network increases, then there is decrease in the end-to-end delay of GPSR because the greedy phase becomes more active. The constraints in GSR for deciding the next hop causes larger delay as compared to E-GyTAR and TFOR. The end-to-end delay of E-GyTAR is more than TFOR because its junction selection mechanism ignores certain rich-density shortest routes and route the packet along longer paths. In addition, the forwarding between junctions based on two-hop neighbor information shortens the route of packet by choosing the closest neighbor to destination that reduces delay. In two-hop neighbor information, packets traverse less number of hops as compared to one-hop neighbor information. Reduced hop count minimizes the end-to-end delay. In improved greedy forwarding, variable speeds of the vehicles induce packet switching, which is more in one-hop neighbor information as compared to two-hop neighbor information. It also increases the end-to-end delay.

The end-to-end delay of the four protocols at 225 vehicular nodes is plotted against different CBR traffics in Figure 10. The end-to-end delay increases with increase in CBR rates, as more packets amplify congestion in the

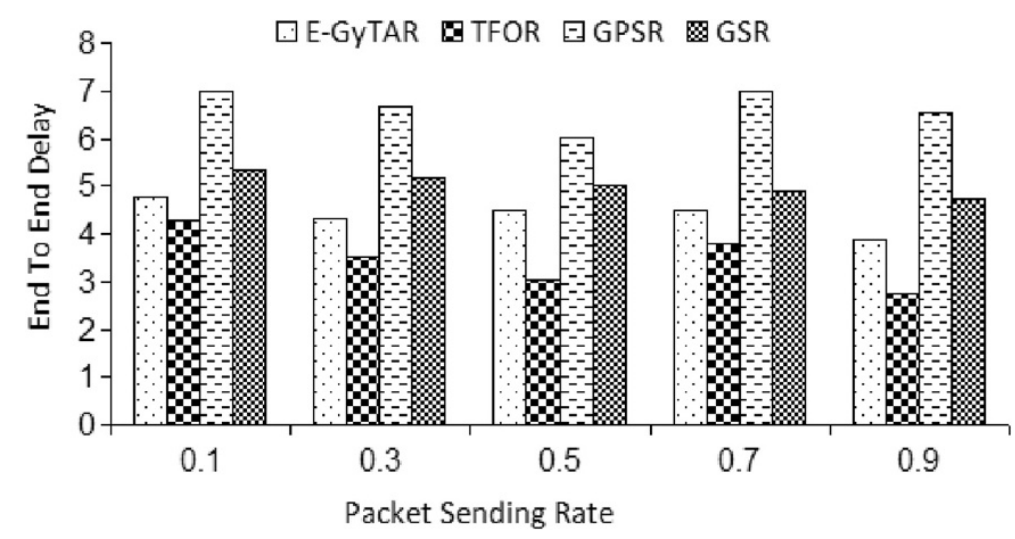

Figure 10 End-to-end delay as a function of the packet sending rate (225 nodes). 


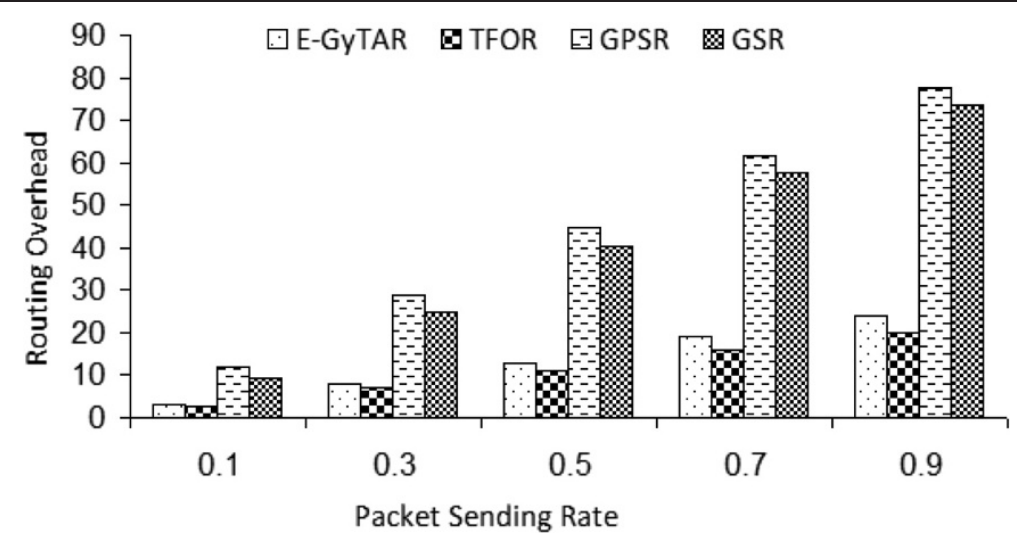

Figure 11 Routing overhead as a function of the packet sending rate (225 nodes).

network. Among these protocols, GPSR has the highest end-to-end delay because of perimeter mode. Due to more control packets transmission, it induces more packets in the network and enhances congestion. The end-to-end delay of GSR is more than that of E-GyTAR and TFOR. The reason is that GSR selects the series of junctions without considering the traffic density. Some city streets do not contain enough vehicles to carry packet from source to destination ensuing the retention of packets in the delay buffer for a longer time, ultimately increasing the end-toend delay. TFOR ensured reduced hop count due to twohop neighbor information. In addition, our simulation confirms that TFOR decides more efficiently the shortest path to the destination as compared to E-GyTAR. Due to these reasons, it has the least end-to-end delay as compared to other routing protocols.

\subsubsection{Routing overhead}

It indicates the ratio of total control packets generated to the total data packets received at the destinations during the complete simulation [24]. Figure 11 plots the routing overhead of all the four protocols against the data-sending rate.

Generally, the routing overhead increases by increasing the packet-sending rate. The routing overhead is the least for all the four protocols at 0.1 packet-sending rate, and steadily, it increases with a raise in the packet-sending rate. The routing overhead of GPSR is the highest as compared to other routing protocols because of its heavy beaconing. GSR exhibits greater routing overhead than TFOR and E-GyTAR (note that the $y$-axis of Figure 11 is on a logarithmic scale). This is because GSR sends higher amounts of beacon messages to acquire the locations of its neighbors. Lochert et al. [8] describe that the number of hello messages that GSR generates are thrice than GyTAR. Among all these, routing protocols TFOR has the minimum routing overhead because of reduced hop count due to two-hop neighbor information.

Figure 12 exemplifies that the growing vehicular density rises routing overhead for both TFOR and E-GyTAR. The fact is that the amount of beacon messages depends on the number of vehicles. The routing overhead of GPSR is the highest because of perimeter mode which causes more transmission of control messages. GSR is the least efficient for its heavy reliance on the control messages to accomplish the location of neighbors. The rest of the protocols are on par with TFOR marginally outperforming the others.

\section{Conclusions}

In this paper, we have provided a brief technical analysis of the existing routing studies along with contribution

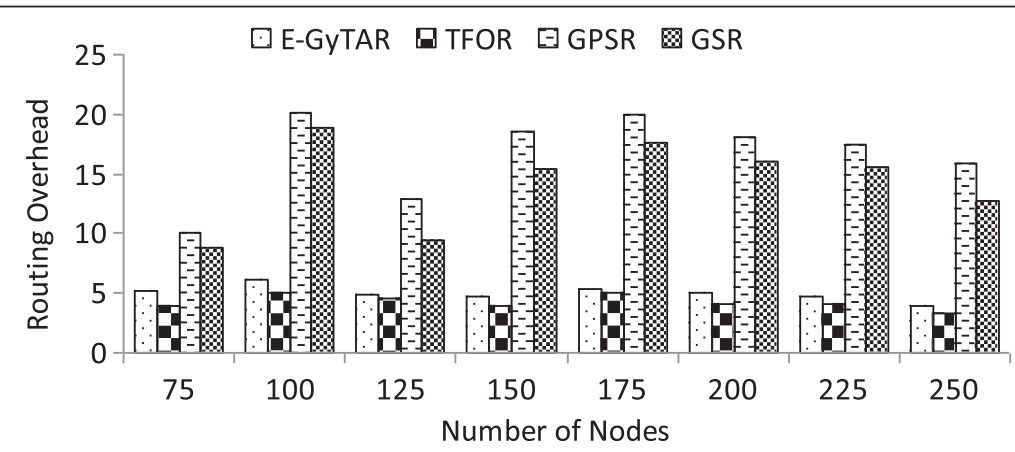

Figure 12 Routing overhead as a function of the number of nodes (at 5 packets/s). 
and comments. We have also proposed, 'traffic floworiented routing (TFOR) protocol', a routing protocol for VANETs. It includes two major phases: first, it selects the next junction optimally, based on directional as well as the non-directional density, and secondly, it uses two-hop neighbor information for routing between the junctions. The comparative study of TFOR with other existing approaches concludes that our routing protocol performs significantly better than the other routing approaches in VANETs. Our simulation outcomes confirm that the TFOR outperforms E-GyTAR, GPSR, and GSR. TFOR performed best in terms of packet-delivery ratio, with an increase of $7.2 \%$ compared to E-GyTAR, more than $16 \%$ as compared to GPSR, and $9 \%$ as compared to GSR. In case of average end-to-end delay, TFOR performed best, with delays of $15.3 \%$ lower than GPSR, $12 \%$ lower than GSR, and $7.5 \%$ lower than E-GyTAR. The proposed improve forwarding mechanism based on two-hop neighbor considerably lowers the average routing overhead as well compared to existing solutions.

In the future, it would be interesting to examine the behavior of TFOR in the presence of one-way roads. A possible research direction could be to design a routing technique that can work in both environments (city and highway).

\section{Competing interests}

The authors declare that they have no competing interests.

\section{Author details}

'Department of Computer Science, King Khalid University (KKU), Abha, Kingdom of Saudi Arabia. ${ }^{2}$ Department of Computer Science, COMSATS Institute of Information Technology (CIIT), Abbotabad 22060, Pakistan. ${ }^{3}$ University Malaysia Sarawak (UNIMAS), Kota Samarahan 94300, Malaysia. ${ }^{4}$ Department of Compute and Information Sciences, Universidad Carlos III de Madrid (UC3M), Leganés 28914, Spain.

Received: 4 February 2014 Accepted: 30 June 2014

Published: 23 July 2014

\section{References}

1. H Hartenstein, KP Laberteaux, VANET Vehicular Applications and Inter-Networking Technologies, 1st edn. (Wiley Online Library, United Kingdom, UK, 2010)

2. S Jaap, M Bechler, L Wolf, Evaluation of routing protocols for vehicular ad hoc networks in city traffic scenarios, in Proceedings of the 5th International Conference on Intelligent Transportation Systems Telecommunications (ITST) (Brest, France, 2005)

3. KC Lee, U Lee, M Gerla, Advances in Vehicular Ad-Hoc Networks: Developments and Challenges, chapter Survey of Routing Protocols in Vehicular Ad Hoc Networks (IGl Global, Hershey, 2009)

4. YW Lin, YS Chen, SL Lee, Routing protocols in vehicular ad hoc networks: a survey and future perspectives. J. Inf. Sci. Eng. 26(3), 913-932 (2010)

5. B Nazir, H Hasbullah, Energy balanced clustering in wireless sensor network, International Symposium in Information Technology (ITSim), 2010, pp. 569-574

6. B Nazir, H Hasbullah, Energy efficient and QoS aware routing protocol for Clustered Wireless Sensor Network. Comput. Electr. Eng. 39(8), 2425-2441 (2013)

7. B Nazir, H Hasbullah, SA Madani, Sleep/wake scheduling scheme for minimizing end-to-end delay in multi-hop wireless sensor networks. EURASIP J. Wirel. Commun. Netw. 92(1), 1-14 (2011)
8. C Lochert, H Hartenstein, J Tian, H Füßler, D Hermann, M Mauve, A routing strategy for vehicular ad hoc networks in city environments, in Proceedings of the Intelligent Vehicles Symposium, 2003. IEEE, 2003, pp. 156-161

9. A Dahiya, RK Chauhan, A comparative study of MANET and VANET environment. J. Comput 2(7), 87-91 (2010)

10. J Gong, CZ Xu, J Holle, Predictive directional greedy routing in vehicular ad hoc networks, in Distributed Computing Systems Workshops, 2007. ICDCSW '07. 27th International Conference on June 2007

11. B Karp, HT Kung, GPSR: greedy perimeter stateless routing for wireless networks, in Proceedings of the 6th annual international conference on Mobile computing and networking, MobiCom 'O0 (ACM, New York, NY, USA, 2000), pp. 243-254

12. F Li, Y Wang, Routing in vehicular ad hoc networks: a survey. Vehicular Technology Maga- zine, IEEE 2(2), 12-22 (2007)

13. A Rahim, I Ahmad, ZS Khan, M Sher, A Javed, M Shoaib, R Mahmood, A comparative study of mobile and vehicular adhoc networks. Int. J. Recent Trends Eng. 2(4), 195-197 (2009)

14. GMT Abdalla, MA Abu-Rgheff, SM Senouci, Current trends in vehicular ad hoc networks. UbiCC Journal, Special Issue on UbiRoads (2008)

15. DB Johnson, DA Maltz, Dynamic source routing in ad hoc wireless networks, in Mobile Computing (Kluwer Academic Publishers, Alphen aan den Rijn, 1996), pp. 153-181

16. CE Perkins, EM Royer, Ad-hoc on-demand distance vector routing, in Mobile Computing Systems and Applications, 1999. Proceedings. WMCSA '99. Second IEEE Workshop, 1999, pp. 90-100

17. T Clausen, P Jacquet, Optimized Link State Routing Protocol (OLSR), RFC 3626 (Internet Engineering Task Force, 2003)

18. AK Gupta, H Sadawarti, AK Verma, Review of various routing protocols for MANETs. Int. J. Inf. Electron. Eng. 1(3), 1-9 (2011)

19. W Kieß, H Fu"ßler, J Widmer, M Mauve, Hierarchical location service for mobile ad-hoc networks: SIGMOBILE Mobile. Comput. Commun. Rev. $1(2), 47-58(2004)$

20. J Li, J Jannotti, DSJ Decouto, DR Karger, R Morris, A scalable location service for geographic ad hoc routing, in Proceedings of the 6th annual international conference on Mobile computing and networking, MobiCom 'OO (ACM, New York, NY, USA, 2000), pp. 120-130

21. M Kasemann, H Füßler, H Hartenstein, M Mauve, A reactive location service for mobile ad hoc networks, Technical Report, TR-14-2002 (Dept. of Computer Science, Univ. of Mannheim, 2002)

22. C Lochert, M Mauve, H Füßler, H Hartenstein, Geographic routing in city scenarios. SIGMOBILE Mob. Comput. Commun. Rev. 9, 69-72 (2005)

23. D Tian, K Shafiee, VCM Leung, Position-based directional vehicular routing, in Proceeding of the Global Telecommunications Conference, GLOBECOM (IEEE, Hoboken, 2009), pp. 1-6

24. M Jerbi, S-M Senouci, R Meraihi, Y Ghamri-Doudane, An improved vehicular ad hoc routing protocol for city environments, in Communications, 2007. ICC '07. IEEE International Conference, 2007, pp. 3972-3979

25. BC Seet, G Liu, BS Lee, CH Foh, KJ Wong, KK Lee, A-STAR, A mobile ad hoc routing strategy for metropolis vehicular communications, in Lecture Notes in Computer Science: NETWORKING 2004, Networking Technologies, Services, and Protocols; Performance of Computer and Communication Networks; Mobile and Wireless Communications, 2004, pp. 989-999

26. S Bilal, SA Madani, IA Khan, Enhanced junction selection mechanism for routing protocol in VANETs. Arab J. Inf. Technol. 8(4), 422-429 (2011)

27. K Prasanth, K Duraiswamy, K Jayasudha, C Chandrasekar, K Prasanth, K Duraiswamy, K Jayasudha, C Chandrasekar, Improved packet forwarding approach in vehicular Ad Hoc networks using RDGR algorithm. International Journal of Next- Generation Networks (IJNGN) 2(1), 64-77 (2010)

28. J Harri, F Filali, C Bonnet, M Fiore, VanetMobiSim: generating realistic mobility patterns for VANETs, in Proceedings of the 3rd international workshop on Vehicular ad hoc networks, VANET'06 (ACM, New York, NY, USA, 2006), pp. 96-97

doi:10.1186/1687-1499-2014-121

Cite this article as: Abbasi et al:: A traffic flow-oriented routing protocol for VANETs. EURASIP Journal on Wireless Communications and Networking 2014 2014:121. 